\title{
Caracterização da má oclusão e do perfil facial de pré-escolares do interior sergipano - um estudo preliminar
}

\section{Characterization of malocclusion and the facial profile of preschool children from upstate Sergipe, Brazil - a preliminary study}

Carla Patrícia Hernandez Alves Ribeiro César Jaqueline Carvalho Silva** Isadora Diniz dos Santos ${ }^{* * *}$ Luanderson Oliveira Silva*** Luiz Renato Paranhos

\section{Resumo}

Anomalias na morfologia facial e na oclusão dental podem acarretar alterações nas funções orais bem como na autoestima, sendo importante que tais problemas sejam detectados o mais precocemente possível para uma adequada intervenção. Objetivo: caracterizar a má oclusão e o perfil facial de pré-escolares do interior do estado de Sergipe. Material e método: em um estudo observacional, de caráter transversal, de forma aleatória, foram selecionadas duas pré-escolas do estado de Sergipe que estivessem atreladas à Diretoria Regional de Educação (DR2), sendo sorteados os municípios de Lagarto e Salgado e eleitas as escolas localizadas no centro. Após apresentação da proposta aos gestores e aos familiares, obteve-se o consentimento para a realização de triagem envolvendo a face e a oclusão. Os resultados obtidos foram submetidos à análise estatística descritiva e ao teste Qui-Quadrado, com relevância adotada de 5\%. Resultados: a idade da amostra variou entre 2:5 e 5:11 (média 4,57 $\pm 1,6$ ), totalizando 86 pré-escolares, desses, $44(51,16 \%)$ do sexo feminino. A maioria dos participantes apresentou perfil convexo (73; 84,88\%), dentadura decídua (62; 72,09\%) e oclusão alterada $(51 ; 59,3 \%)$. Não foi encontrado resultado estatisticamente significativo entre as dentaduras e o perfil facial. Conclusão: o perfil convexo prevaleceu como características de pré-escolares do interior sergipano. Os pré-escolares apresentaram alterações na oclusão, revelando necessidade de intervenção precoce. É necessária a implantação do serviço ortodôntico nos municípios envolvidos na pesquisa.

Palavras-chave: Face. Má oclusão. Pré-escolar.

\section{Introdução}

Os conhecimentos da morfologia facial e da sua relação com a oclusão são prerrogativas para o correto diagnóstico e o estabelecimento de metas terapêuticas individualizadas ${ }^{1}$. Para tal, faz-se necessária a compreensão do processo do crescimento craniofacial, que se dá desde sua forma embriológica até a maturidade esquelética, passando pela infância e pela adolescência ${ }^{2}$. Anormalidades congênitas, hereditárias e ambientais, em diferentes fases da vida, podem acarretar desordens oclusais ${ }^{3}$.

A cárie e as doenças periodontais são os principais problemas de saúde bucal ${ }^{4}$, e, epidemiologicamente, os problemas de oclusão apresentam-se como o terceiro maior problema bucal, sendo, dessa forma, elencados como prioridades relacionadas aos problemas odontológicos de saúde pública ${ }^{5}$.

Problemas oclusais, como mordida aberta anterior, geralmente, são diagnosticados na fase da dentadura decídua e mista, época que coincide com o desenvolvimento craniofacial. Quando esses problemas são interceptados precocemente, as chances de sucesso no tratamento são maiores ${ }^{6}$. As alterações oclusais podem se apresentar em diferentes níveis de gravidade, que, consequentemente, receberão diferentes prioridades de tratamento. Quanto mais rápido o diagnóstico, melhor o prognóstico, evitando, assim, agravos e possíveis evoluções cirúrgicas ${ }^{7}$. 
Os distúrbios de fala, do modo respiratório ${ }^{4}$, da mastigação ${ }^{8} \mathrm{e}$ da deglutição $0^{4,8}$ são os problemas mais comuns em pré-escolares. Respiradores predominantemente bucais tendem a apresentar dificuldades no aprendizado, interferindo negativamente no rendimento escolar ${ }^{9}$.

Em relação à fonoaudiologia, apesar de ser reconhecida como profissão que exige nível superior de graduação desde 1981, as práticas no âmbito escolar são recentes e necessitam de maior reforço ${ }^{10}$. São importantes a criação e o incentivo de práticas comunitárias que promovam o crescimento da consciência sanitária e a mobilização da sociedade relacionada às questões de saúde ${ }^{11}$, que podem e devem ser alicerçadas pelo conhecimento epidemiológico da situação da população, a fim de serem efetivadas ações de planejamento, desde a prevenção até o tratamento ${ }^{3}$, sendo importante a parceria entre a Odontologia e a Fonoaudiologia.

Diante do exposto, percebe-se que a análise do perfil facial e de sua relação com a oclusão é eficaz e importante no processo de diagnóstico, nas tomadas de decisões sobre os tratamentos odontológicos e fonoaudiológicos e nas análises epidemiológicas da saúde bucal. Assim, este trabalho teve por objetivo caracterizar a má oclusão e o perfil facial de pré-escolares do interior do estado de Sergipe, como medida auxiliar na implementação de políticas públicas.

\section{Material e método}

Este estudo foi previamente aprovado pelo Comitê de Ética em Pesquisa com Seres Humanos (CAAE: 0060.0.214.000-09), cumprindo com o determinado na Resolução do Conselho Nacional de Saúde no 466/2012, de 12 de dezembro de 2012.

Trata-se de um estudo observacional, de caráter transversal e descritivo, realizado em pré-escolares do estado do Sergipe (Nordeste brasileiro). Segundo os dados do Instituto Brasileiro de Geografia e Estatística (IBGE), a população estimada de Sergipe, em 2016, era de 2.265.779 habitantes, distribuídos em 75 municípios. Em 2015, o rendimento nominal mensal domiciliar per capita da população residente era de $\mathrm{R} \$ 782,00^{12}$.

Para execução deste estudo preliminar, foram escolhidas aleatoriamente (por sorteio) duas escolas que estivessem atreladas à Diretoria Regional de Educação (DR2), compreendendo as cidades de Lagarto, Salgado, Boquim, Poço Verde, Simão Dias e Riachão do Dantas, as sorteadas foram Lagarto e Salgado.

Os municípios de Lagarto e Salgado, localizados no centro-sul do estado de Sergipe, ficam próximos entre si $(23,8 \mathrm{~km})$, sendo que Lagarto apresenta área aproximadamente quatro vezes maior $\left(1.036 \mathrm{~km}^{2}\right)$ do que Salgado $\left(255,8 \mathrm{~km}^{2}\right)$. Em termos populacionais, Lagarto e Salgado, juntos, representam aproximadamente $5,5 \%$ dos habitantes do estado. Lagarto contempla 21 instituições educacio- nais (12 públicas e 9 privadas), enquanto Salgado contempla 8 (4 públicas e 4 privadas $)^{12}$. Lagarto é uma cidade com 103.188 habitantes e Salgado, com 20.126 habitantes, segundo estimativas ${ }^{12}$.

Após contato com a Secretaria de Educação dos municípios, com os gestores das escolas e os responsáveis, foram avaliados os pré-escolares a partir dos critérios elegíveis escolhidos.

Foram incluídos no estudo pré-escolares entre 2 e 6 anos de idade, portadores de autorização dos pais e dos educadores para participar da triagem. Como critério de exclusão, os pré-escolares não poderiam: estar em (ou ter efetivado) tratamento fonoaudiológico, ortodôntico ou ortopédico facial; ter histórico positivo de cirurgias (faciais e faríngeas); ter queixas (familiares ou dos educadores) de alterações neurológicas, genéticas, malformações craniofaciais, intelectuais ou auditivas que pudessem comprometer a compreensão das instruções fornecidas; e estar resfriados ou gripados no dia do teste.

Para a realização da triagem da oclusão e da face, dois profissionais especialistas e com mais de vinte anos de experiência em suas áreas (Odontologia e Fonoaudiologia), denominados como pesquisadores instrutores, realizaram a capacitação de oito horas para os demais pesquisadores, sendo as primeiras quatro horas destinadas aos aspectos teórico-práticos e as quatro restantes para atividade prática supervisionada, com a realização das triagens e posterior discussão. Determinou-se um pesquisador padrão, ou seja, que apresentou menos divergências entre suas constatações com as dos pesquisadores instrutores, para acompanhar todas as triagens realizadas.

O instrumento para coleta de dados foi composto por duas partes. A primeira com informações relacionadas à identificação do pré-escolar (nome, idade, série, dados maternos e paternos, como: nome, escolaridade e renda familiar) e com dados relativos à triagem, como a análise subjetiva e clínica de perfil facial (côncavo, convexo ou reto), dentes (quantidade, dentadura, falhas dentais e saúde oral) e oclusão (linha média adequada ou desviada, com a possibilidade de se indicar o lado direito ou esquerdo), oclusão normal ou alterada, ausência ou presença de trespasse horizontal - TH (mordida de topo: $\mathrm{TH}=0 \mathrm{~mm}$, sobressaliência: $\mathrm{TH}>3 \mathrm{~mm}$ ou mordida cruzada anterior: $\mathrm{TH}<0 \mathrm{~mm}$ ), vertical - TV (mordida de topo: TV=0 mm, sobremordida: TV $>3 \mathrm{~mm}$, mordida aberta anterior ou posterior). Foi incluída na avaliação a observação da relação entre os arcos (maxilar e mandibular) e dos caninos. Após a inspeção visual, foi realizada tomada fotográfica para confirmação da triagem tanto da face quanto da oclusão.

Conforme apontado pela literatura ${ }^{13}$, para um correto processo de observação, é importante a realização da documentação por meio de registro de imagens estáticas (por captura fotográfica), atentando-se para os devidos cuidados, tais como: indivíduo 
em pé, com braços soltos ao longo do corpo, próximo a uma parede lisa e sem brilho (evitando apoiar-se na parede) e descalço; utilização de câmera com boa resolução (máquina fotográfica Sony Cyber Shot, digital, 7,2 megapixels, modelo DSC P200), apoiada em um tripé; boa iluminação da sala; e uso de afastadores labiais para os registros intraorais.

As triagens foram realizadas em salas cedidas pelas instituições de ensino, individualmente, por tempo estimado de 20 minutos por criança. A partir dos resultados obtidos, os familiares e os gestores receberam devolutivas e foram orientados quanto às condutas para discussão de ações necessárias para a diminuição das alterações existentes.

Os resultados obtidos foram digitados no software de planilha eletrônica Excel (pacote Microsoft ${ }^{\circledR}$ Office). Os pré-escolares foram identificados por números, tendo como base de dados o nome da instituição de educação infantil, a data da triagem, a idade da criança, o sexo, o perfil facial, o tipo de dentadura e linha média. Foi utilizada análise estatística descritiva a partir das variáveis de análise: alterações horizontais, verticais e transversais. Para a comparação entre os grupos, foi utilizado o teste Qui-Quadrado, considerando-se valor de $\mathrm{p}$ de $5 \%$ como relevante para o estudo.

\section{Resulltados}

A idade da amostra variou entre 2 anos e 5 meses e 5 anos e 11 meses (média: 4,57 \pm 1,6), sendo 44 $(51,16 \%)$ do sexo feminino e $42(48,84 \%)$ do masculino. A amostra foi composta por 86 pré-escolares dos municípios de Lagarto e Salgado, do estado de Sergipe.

A maioria dos participantes apresentou perfil convexo $(n=73 ; 84,88 \%)$, dentadura decídua $(n=62$; $72,09 \%)$, linha média adequada $(n=55 ; 63,95 \%)$ e oclusão alterada $(\mathrm{n}=51 ; 59,3 \%)$.

$\mathrm{Na}$ Tabela 1, podem ser observadas as alterações horizontais, verticais e transversais obtidas neste estudo e, na Tabela 2, a comparação entre os perfis e as dentaduras, não sendo revelados valores estatisticamente significantes.

Tabela 1 - Análise das dimensões horizontais, verticais e transversais da oclusão de 86 pré-escolares do estado de Sergipe, Brasil

\begin{tabular}{l|l|r|r}
\hline \multicolumn{1}{c|}{ Alterações } & $\mathrm{N}$ & \multicolumn{1}{c}{$\%$} \\
\hline \multirow{4}{*}{ Horizontal } & Ausente & 60 & 69,76 \\
& TH=0 mm & 13 & 15,11 \\
& TH>3 mm & 9 & 10,46 \\
& TH $<0 \mathrm{~mm}$ & 4 & 4,65 \\
\multirow{5}{*}{ Vertical } & Ausente & 55 & 63,95 \\
& TV=0 mm & 6 & 6,97 \\
& TV>3 mm & 15 & 17,44 \\
Transversal & TV $<0 \mathrm{~mm}$ & 10 & 11,62 \\
& Mordida aberta posterior & 0 & 0 \\
& Ausente & 76 & 88,37 \\
& Mordida cruzada posterior D & 5 & 5,81 \\
& Mordida cruzada posterior E & 5 & 5,81 \\
\hline
\end{tabular}

Fonte: elaboração dos autores.

$\mathrm{TH}=$ trespasse horizontal; $\mathrm{TV}=$ trespasse vertical; $\mathrm{D}=$ direita; $\mathrm{E}=$ esquerda; e $\mathrm{N}$ = número de sujeitos.
Tabela 2 - Comparação entre a análise facial e as dentaduras decídua e mista dos 86 pré-escolares do estudo pelo teste Qui-Quadrado

\begin{tabular}{c|c|c|c|c}
\hline \multicolumn{5}{|c}{ Perfil facial } \\
\hline Dentadura & Côncavo & Convexo & Reto & Total \\
\hline Decídua & 5 & 52 & 5 & 62 \\
Mista & 0 & 21 & 3 & 24 \\
Total & 5 & 73 & 8 & 86 \\
\hline \multicolumn{5}{|c}{$\mathrm{p}=0,312$} \\
\hline
\end{tabular}

Fonte: elaboração dos autores.

\section{Discussão}

Este estudo foi centrado nos aspectos oclusais e no perfil facial de pré-escolares. Para a atuação profissional em Fonoaudiologia e em Odontologia, são importantes o conhecimento do desenvolvimento do complexo crânio-oro-cervical e a compreensão de suas inter-relações, favorecendo a implantação de medidas interventivas ${ }^{1,14}$, as quais, quando inseridas no âmbito escolar, têm o cunho preventivo, principalmente porque podem favorecer a obtenção da harmonia entre as estruturas e as funções estomatognáticas - importantes para a comunicação interpessoal, a sobrevivência e a estética.

Dessa forma, dimensionar as características de pré-escolares quanto aos aspectos faciais e oclusais é uma tarefa importante e necessária para o planejamento de ações individuais e coletivas na educação, promovendo a reflexão de medidas em saúde que possam minimizar ou até mesmo sanar as alterações existentes ${ }^{3,4}$, dependendo da gravidade ${ }^{11} \mathrm{e}$ do engajamento dos envolvidos ${ }^{7}$. Tais medidas podem abranger desde precauções de doenças cariogênicas, periodontais $^{4}$ e oclusais ${ }^{8}$ até medidas para prevenir as alterações do sistema estomatognático ${ }^{4,8}$, requerendo um papel proativo dos profissionais da saúde, além do engajamento político para a implementação de políticas públicas ${ }^{4}$.

Um dos resultados obtidos neste estudo foi a adequação da linha média na maioria dos pré-escolares $(63,95 \%)$, evidenciando harmonia esquelética entre os arcos dentais e a face. O desvio da linha média facial com a dental, na infância, pode ocorrer por diversos motivos e sua detecção possibilita o encaminhamento precoce para diversos especialistas. Na odontologia, os profissionais investigam se o desalinhamento é dental ou esquelético, procedendo com suas devidas condutas; na fonoaudiologia, investigam se há ou não alterações na respiração, mastigação, deglutição e fala; e na otorrinolaringologia, verificam o tamanho das tonsilas (palatinas e faríngea), se há ou não desvio de septo nasal, entre outros.

As alterações transversais também foram observadas em baixos percentuais entre os pré-escolares triados (12,78\%), verificando-se que alguns pré-escolares apresentaram desvio funcional da mandíbula, observado por meio do posicionamento da mandíbula em relação central (RC) e com os den- 
tes em máxima intercuspidação (MIC). A explicação de tal desvio funcional pode ocorrer, segundo a literatura ${ }^{15}$, por interferências oclusais durante os movimentos funcionais. Os desvios oclusais podem interferir negativamente na função mastigatória ${ }^{16}$, e, em decorrência disso, alguns comportamentos musculares compensatórios podem também desencadear distúrbios miofuncionais orofaciais ${ }^{17}$.

A maioria dos pré-escolares apresentou má oclusão dental (59,3\%), com alterações isoladas ou combinadas nas relações verticais $(36,05 \%)$, horizontais $(31,4 \%)$ e transversais $(11,63 \%)$ entre os arcos dentais. Esses resultados se assemelham aos encontrados na literatura ${ }^{18,19}$. Kasparaviciene et al. ${ }^{19}(2014)$ obtiveram a prevalência de má oclusão de $71,4 \%$ entre os pré-escolares com idade entre 5 e 7 anos. Esse mesmo estudo ${ }^{19}$ associou os hábitos de sucção com a mordida aberta anterior e mordida cruzada posterior, destacando que a interposição anterior da língua ao deglutir pode ser uma provável causa do desenvolvimento da mordida aberta anterior.

Outros pesquisadores ${ }^{20}$ associaram aspectos respiratórios (como a presença de dor de garganta, resfriados constantes, bronquite, alergia e sinusite) em $93,2 \%$ das crianças $(\mathrm{n}=275)$ entre 4 e 6 anos de idade estudadas, sendo que essas crianças permaneciam com a boca aberta durante a noite e apresentavam hábitos orais. Em um estudo ${ }^{21}$ realizado com 176 crianças com a mesma faixa etária deste trabalho, analisou-se uma possível associação entre o hábito de sucção não nutritiva, mordida aberta anterior e perfil facial, concluindo que esse hábito, durante a fase de dentição decídua, influencia a má oclusão e a mordida aberta anterior - independente do perfil facial, corroborando os achados desta pesquisa.

Com relação ao perfil facial, na comparação entre a análise facial e as dentaduras decídua e mista, não foram revelados valores estatisticamente significativos. No entanto, vale observar que a maioria da amostra do estudo tinha um perfil convexo, independente da dentadura. Pesquisadores ${ }^{22}$ também concluíram que o perfil convexo foi o mais prevalente em crianças da mesma faixa etária que as deste estudo.

Diferente do que foi encontrado em estudo realizado em outro estado ${ }^{23}$, que apresentou, nos seus resultados, uma predominância do padrão facial tipo I (reto) em uma amostra constituída por 875 crianças, com idade média de 6,7 anos, em relação ao perfil facial, no presente estudo, foi constatado perfil convexo para a maioria da amostra $(84,88 \%)$. Este fato deve ser levado em consideração, uma vez que a maioria dos indivíduos apresenta um perfil convexo ao nascimento e tem o padrão estabelecido definitivamente perto do surto puberal. $\mathrm{O}$ perfil facial tipo II (convexo) se caracteriza por um degrau sagital positivo entre a maxila e a mandíbula ${ }^{14}$. Um estudo indiano ${ }^{24}$ constatou associação entre este tipo de perfil com a relação plana e com a distal dos segundos molares decíduos.

A análise clínica e subjetiva da face e da oclusão é tão importante quanto a realizada por meio de imagens, e a inclusão da análise frontal com a do perfil da face em triagens é um procedimento simples e de fácil aplicação, que permite prever futuras assimetrias ou anomalias no decorrer do crescimento facial infantil. Assim, medidas profiláticas e interceptativas podem ser aplicadas para minimizar as possíveis sequelas ao sistema estomatognático, como no desempenho deficitário das funções orais (fala, mastigação e deglutição), nas más oclusões e na desarmonia musculoesquelética facial.

Com relação à mordida cruzada posterior, ela esteve presente em $11,62 \%$ dos pré-escolares desta pesquisa; valor próximo do observado por outros pesquisadores ${ }^{25}$, que constataram sua presença em 10 crianças $(20,41 \%)$ com dentição decídua. Tal manifestação pode afetar a mastigação, podendo haver diminuição da velocidade ${ }^{25}$ e da força na mastigação ${ }^{26}$, bem como crescimento e desenvolvimento craniofacial ${ }^{27,28}$, reforçando os benefícios da intervenção precoce. Uma revisão de literatura ${ }^{29}$ realizada em 2016 concluiu que mordida cruzada posterior apresenta alta prevalência, podendo estar relacionada à presença de hábitos bucais como sucção e respiração oral, resultando em um desenvolvimento anormal dos maxilares e, em consequência, em um desarranjo oclusal.

Uma limitação deste estudo refere-se ao tamanho da amostra, no entanto, cabe ressaltar que esta pesquisa teve um caráter preliminar, tendo em vista a aplicação de um instrumento de coleta de dados por duas especialidades: a Odontologia e a Fonoaudiologia. Dessa forma, houve a necessidade de aproximação de docentes das duas áreas (ortodontia e motricidade orofacial, respectivamente), para elaboração de um protocolo único de triagem e para a criação de uma capacitação, resultando em espaços interdisciplinares de reflexão tanto sobre os saberes quanto sobre as condutas a serem tomadas. Essa parceria interprofissional favorece a geração de recursos humanos em saúde mais capacitados e, segundo a literatura ${ }^{30}$, pode potencializar ações promotoras e preventivas em saúde. Além disso, mais estudos acerca da temática abordada são necessários e fazem-se importantes para o enriquecimento da literatura sobre o assunto.

Outro aspecto a ser observado é que os indivíduos estudados estão com a oclusão se estabelecendo, e os problemas oclusais adquiridos nessa época evidenciam a necessidade de intervenções terapêuticas, como ortodontia preventiva e interceptadora, promovendo também a harmonia da execução das funções do sistema estomatognático, como a fala, a deglutição e a mastigação. Levantamentos epidemiológicos em fases precoces da vida são necessários para o oferecimento de condutas que possam 
sanar as alterações encontradas, bem como para a cobrança, às instâncias públicas, da garantia de tratamento ortodôntico preventivo e interceptativo nos municípios do interior do estado de Sergipe.

\section{Conclusão}

Com base nos resultados do presente estudo, pode-se concluir que o perfil convexo prevaleceu como característica de pré-escolares do interior sergipano, no entanto, mais da metade dos pré-escolares apresentou alterações na oclusão, revelando as necessidades de intervenção precoce e implantação do serviço ortodôntico nos municípios envolvidos na pesquisa.

\section{Abstract}

Anomalies in facial morphology and dental occlusion may result in changes of both oral functions and self-esteem, and it is important for such issues to be detected as early as possible for a proper intervention. Objective: to characterize malocclusion and the profile of preschool children from upstate Sergipe, Brazil. Materials and method: it was a cross-sectional observational study, which randomly selected two preschools of the state of Sergipe, Brazil that were linked to the Regional Board of Education (DR2). The cities of Lagarto and Salgado were drawn and the schools selected were located in the downtown area of these cities. After presenting the proposal to school managers and family members, they consented to perform the triage involving face and occlusion. The results obtained were subjected to descriptive statistical analysis and to the chi-square test at 5\% significance. Results: the age of the sample ranged from

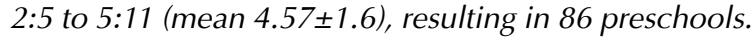
From the sample, $44(51.16 \%)$ were girls. Most of the participants presented a convex profile $(73 ; 84.88 \%)$, deciduous teeth $(62 ; 72.09 \%)$, and changed occlusion $(51 ; 59.3 \%)$. There were no statistically significant results between teeth and the facial profile. Conclusion: the convex profile prevailed as a characteristic of preschool children from upstate Sergipe, Brazil. The preschool children presented changes in occlusion, showing the need for early intervention. The implementation of orthodontic care is required in the cities involved in the research.

Keywords: Face. Malocclusion. Preschool children.

\section{Referências}

1. Capelozza Filho L. Diagnóstico em ortodontia. Maringá: Dental Press; 2012.

2. Silva Filho OG, Queiroz APC, Herkrath FJ, Silva GFB. Correlação entre padrão facial e relação sagital entre os arcos dentários no estágio de dentadura decídua: considerações epidemiológicas. R Dental Press Ortodon Ortop Facial 2008; 13(1):101-12.

3. Alves JAO, Forte FDS, Sampaio FC. Condição socioeconômica e prevalência de más oclusões em crianças de 5 e 12 anos na USF Castelo Branco III - João Pessoa/Paraíba. R Dental Press Ortodon Ortop Facial 2009; 14(3):52-9.
4. Verrastro AP, Tashima AY, Ideriha PN, Stefani FM, Rodrigues CRMD, Wanderley MT. Características oclusais e miofuncionais orais das crianças atendidas na Clínica de Odontopediatria da Faculdade de Odontologia da USP. Rev Inst Ciênc Saúde 2009; 27(4):394-9.

5. Higiene dental: reunión de un grupo de consultores de la OMS. Cronica de la OMS 1955; 9:11-6.

6. Nakao TH, Berto FA, De Oliveira DTN, Berto APM, Bigliazzi R. Hábitos bucais como fatores de risco para a mordida aberta anterior: uma revisão de literatura. Revista Odontológica de Araçatuba 2016; 37(2):9-16.

7. Pauleto ARC, Pereira MLT, Cyrino EG. Saúde bucal: uma revisão crítica sobre programações educativas para escolares. Ciência \& Saúde Coletiva 2004; 9(1):121-30.

8. Maciel KRA, Albino RCM, Pinto MMA. A prevalência de distúrbio miofuncional orofacial nos pacientes atendidos no ambulatório de pediatria do Hospital Luís de França. Rev Pediatr 2007; 8(2):81-90.

9. Ribeiro GCA, dos Santos ID, Santos ACN, Paranhos LR, César CPHAR. Influence of the breathing pattern on the learning process: a systematic review of literature. Braz J Otorhinolaryngol 2016; 82(4):466-78.

10. Maranhão PCS, Pinto SMPC, Pedruzzi CM. Fonoaudiologia e educação infantil: uma parceria necessária. Rev Cefac 2009; 11(1):59-66.

11. Boeck EM, Pizzol KEDC, Navarro N, Chiozzini NM, Foschini ALR. Prevalência de maloclusão em escolares de 5 a 12 anos de rede municipal de ensino de Araraquara. Rev Cefac 2013; 15(5):1270-80.

12. Instituto Brasileiro de Geografia e Estatística. Estados@. Rio de Janeiro: IBGE; 2010. [Acesso em 08 set. 2017]. Disponível em URL: http://www.ibge.gov.br/estadosat/perfil.php?sigla=se.

13. Genaro KF, Felício CM. Protocolos clínicos de avaliação miofuncional orofacial. In: Marchesan IQ, Silva HJ, Tomé MC (Org.). Tratado das especialidades em Fonoaudiologia. São Paulo: Guanabara-Koogan; 2014. p. 271-82.

14. Silva Filho OG, Herkrath FJ, Queiroz APC, Aiello CA. Padrão facial na dentadura decídua: estudo epidemiológico. $\mathrm{R}$ Dental Press Ortodon Ortop Facial 2008; 13(4):45-59.

15. Chibinski ACR, Czlusniak GD, Melo MDD. Pistas diretas planas: terapia ortopédica para correção de mordida cruzada funcional. R Clin Ortodon Dental Press 2005; 4:64-72.

16. Maltagliati LA, Montes LAP, Bastia FMM, Bommarito S. Avaliação da prevalência das seis chaves de oclusão de Andrews, em jovens brasileiros com oclusão normal natural. $R$ Dental Press Ortodon Ortop Facial 2006; 11(1):99-106.

17. Chiodelli L, Pacheco AB, Missau TS, Silva AMT, Corrêa ECR. Associação entre funções estomatognáticas, oclusão dentária e sinais de disfuncão temporomandibular em mulheres assintomáticas. Rev Cefac 2015; 17(1):117-25.

18. Katz CRT, Rosenblatt A, Gondim PPC. Hábitos de sucção, padrão de crescimento facial e alterações oclusais em pré-escolares do Recife - PE. J Bras Ortodon Ortop Facial 2002; 7(40):306-13.

19. Kasparaviciene K, Sidlauskas A, Zasciurinskiene E, Vasiliauskas A, Juodzbalys G, Sidlauskas M, et al. The prevalence of malocclusion and oral habits among 5-7-year-old children. Med Sci Monit 2014; 20:2036-42.

20. Lima GN, Cordeiro CM, Justo JS, Rodrigues LCB. Mordida aberta anterior e hábitos orais em crianças. Rev Soc Bras Fonoaudiol 2010; 15(3):369-75.

21. Fialho MPN. Estudo da associação entre hábito de sucção não nutritiva, mordida aberta anterior e morfologia facial [Dissertação de Mestrado]. São Luís: Universidade Ceuma; 2012. 
22. Souza DFRK, Valle MAS, Pacheco MCT. Relação clínica entre hábitos de sucção, má oclusão, aleitamento e grau de informação prévia das mães. Revista Dental Press de Ortodontia e Ortopedia Facial 2006; 11(6):81-90.

23. Traldi A, Valdrighi HC, Souza LZ, Vedovello SAS. Evaluation of facial morphology and sagittal relationship between dental arches in primary and mixed dentition. Dental Press J Orthod 2015; 20(4):63-7.

24. Sherene A, Neeta TP. Profiles, occlusal plane relationships and spacing of teeth in the dentitions of 3 to 4-year-old children. J Clin Pediatr Dent 1998; 22(4):329-34.

25. Throckmorton GS, Buschang PH, Hayasaki H, Pinto AS. Changes in the masticatory cycle following treatment of posterior unilateral crossbite in children. Am J Orthod Dentofacial Orthop 2001; 120(5):521-9.

26. Sonnesen L, Bakke M, Solow B. Bite force in pre-orthodontic children with unilateral crossbite. Eur J Orthod 2001; 23(6):741-9.

27. Castelo PM, Bonjardim LR, Pereira LJ, Gavião MBD. Facial dimensions, bite force and masticatory muscle thickness in preschool children with functional posterior crossbite. Braz Oral Res 2008; 22(1):48-54.

28. Gavião MBD, Chelotti A. Estudo comparativo dos arcos dentários decíduos espaçados e não espaçados: segmento anterior. Rev Odontopediatr 1992; 1(3):195-201.

29. Batista ER, dos Santos DCL. Mordida cruzada posterior em dentição mista. Rev Odontol Univ Cid São Paulo 2016; 29(1):66-74.

30. Luchesi KF, Toledo IP, Vieira AS, Meurer BE, Quadros DI, Corso MT, et al. Fonoaudiologia e Odontologia na atenção básica: relato de experiência de educação em saúde. Distúrbios da Comunicação 2016; 28(2):388-93.

\section{Endereço para correspondência:}

Carla Patrícia Hernandez Alves Ribeiro César

Rua Laudelino Freire, 184, $2^{\circ}$ andar, Centro

CEP 49400-000 Lagarto, SE, Brasil

Telefone: (51) 939537140

E-mail: carlacesar@globo.com

Recebido: 10/11/17. Aceito: 04/12/17. 